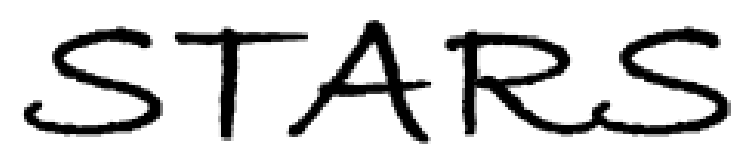

University of Central Florida

STARS

Faculty Bibliography 1990s

Faculty Bibliography

$1-1-1997$

\title{
Dynamics of low-dimensional dipolar systems
}

\author{
J. M. Sun \\ University of Central Florida \\ Weili Luo \\ University of Central Florida
}

Find similar works at: https://stars.library.ucf.edu/facultybib1990

University of Central Florida Libraries http://library.ucf.edu

This Article is brought to you for free and open access by the Faculty Bibliography at STARS. It has been accepted for inclusion in Faculty Bibliography 1990s by an authorized administrator of STARS. For more information, please contactSTARS@ucf.edu.

\section{Recommended Citation}

Sun, J. M. and Luo, Weili, "Dynamics of low-dimensional dipolar systems" (1997). Faculty Bibliography 1990s. 2106.

https://stars.library.ucf.edu/facultybib1990/2106

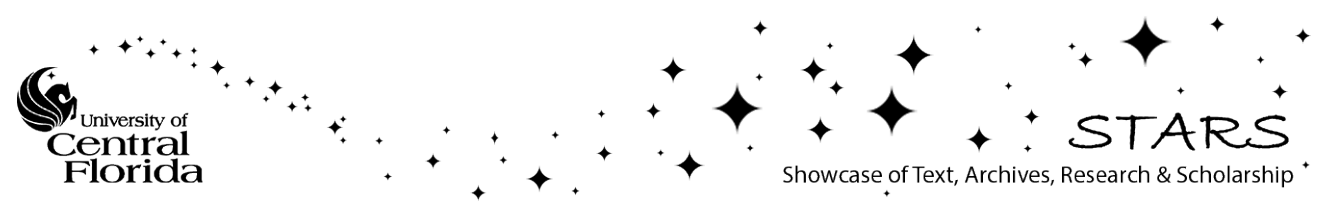




\title{
Dynamics of low-dimensional dipolar systems
}

\author{
J. M. Sun* and Weili Luo ${ }^{\dagger}$ \\ Department of Physics, University of Central Florida, Orlando, Florida 32816
}

(Received 9 April 1997; revised manuscript received 1 July 1997)

\begin{abstract}
Time decays of low-dimensional magnetic dipoles with anisotropic energy barriers were studied theoretically. We found that the dynamic behaviors are interaction specific. For a chain with ferromagnetic coupling, a single mode dominates and the decay is essentially exponential. For antiferromagnetic coupling, the dynamics can be characterized by two distinct groups of time scales. The spectrum results in a plateau in the decay of remanent magnetization. This novel behavior differs from the prediction of the mean-field theory. For a two-dimensional rectangular lattice, a transition between the bimodal decay and a quasilogarithmic decay occurs when the ratio of length to width varies. [S1063-651X(97)06310-1]

PACS number(s): $02.50 .-\mathrm{r}$
\end{abstract}

\section{INTRODUCTION}

Although slow relaxation is a universal phenomenon in complex systems such as glasses [1,2], spin glasses [3,4], and disordered materials in general, microscopic mechanisms that govern general features observed in diverse systems are still poorly understood. Recent experiments on the dynamic susceptibilities [5] and magnetizations [6] of frozen ferrofluids demonstrated that the dipole interaction between magnetic particles has a dramatic influence on the dynamics of the system and the effect might be accounted for by simple laws. Inspired by this work, it is our motivation to study dynamics based on similar dipolar systems.

For a noninteracting system, the decay of a physical quantity $q(t)$ towards equilibrium can be described by a simple exponential [7]. The time scale of the decay is described by a relaxation time $\tau$. It has been suggested that the nonexponential decay is associated with the frustrated interaction and disorder, which leads to a broad distribution of relaxation times [8]. Taking this distribution into account, the time dependence can be described by

$$
q(t)=\int_{0}^{\infty} w(\tau) \exp (-t / \tau) d \tau
$$

where $w(\tau)$ is the distribution function for $\tau$. To incorporate the effect of correlation in strong interacting systems, a hierarchy among relaxation channels has been introduced [9]. However, since such an approach is phenomenological in nature, it provides no obvious link to the microscopic origin of the distribution, $w(\tau)$.

Microscopic understanding of the cooperative dynamics can be achieved through the study of various models [10-14] which usually introduce specific transition rates. For example, in dynamics for the Ising model, the transition rate for a spin $\sigma$ to flip into $-\sigma$ is chosen as $[10,14]$

\footnotetext{
*Present address: Physics Department, Florida International University, University Park, Miami, FL 33199.

†Electronic address: luo@pegasus.cc.ucf.edu
}

$$
W(\sigma \rightarrow-\sigma)=\frac{1}{2 \tau_{0}}[1-\sigma \tanh (\beta H)],
$$

where $\tau_{0}$ is a characteristic time scale, $\beta$ the Boltzmann factor, and $H$ the effective field on the spin $\sigma$. Glauber has succeeded in solving the one-dimensional case of Eq. (2) for quantum spins exactly and great insight is obtained about the complex cooperative dynamics in interacting systems [10].

The choice of transition rate depends on the specific system studied. For classical system with an anisotropy barrier, Néel's flipping transition rate [15] is often used:

$$
W(\sigma \rightarrow-\sigma)=\frac{1}{\tau_{0}} \exp \left[-\beta E_{b}\left(1+\frac{\sigma H}{2 E_{b}}\right)^{2}\right],
$$

where $E_{b}$ is the activation barrier. Equation (3) is well known in the study of the relaxation process of magnetic fine particle systems and has recently been used in the investigation of magnetic remanence of perpendicular media [16-19]. A mean-field version of Eq. (3), with no disorder assumed, is able to reproduce the quasilogarithmic decay observed in perpendicular film media [17]. This is a clear indication that a broad spectrum of time scales, as implied by quasilogarithmic law, can exist in a uniform interacting system.

The present work addresses the dynamics of onedimensional (1D) dipole rings based on Néel's theory of flipping transition in Eq. (3). For antiferromagnetic coupling, the model displays a novel bimodal relaxation, characteristically different from the prediction of mean-field theory. Although not reported experimentally yet, we stress that the phenomena are observable in real perpendicular media under proper conditions, as demonstrated by our Monte Carlo simulations for the film geometry. In Sec. II we will describe our model and methods. Our model is based on a real system of superparamagnetic fine particles similar to the ones in Ref. [5]. The system had been suggested as a model for studying the glass transition [20]. The geometric arrangements include ferromagnetic chains with periodic boundary condition, antiferromagnetic rings in which the spin orientations are perpendicular to the plane on which they reside, and films of rectangular lattices with antiferromagnetic couplings. The methods involve solving eigenproblems for smaller systems and dynamic Monte Carlo simulations on large systems. The 
discussion of the results will be presented in Sec. III. Our calculations indicate that for a chain structure, unlike the Glauber model where the total magnetization always decays exponentially, a distinction exists between ferromagnetic and antiferromagnetic chains. While ferromagnetic chains display a quasiexponential decay of remanence, the antiferromagnetic chains show a more complex behavior. At large antiferromagnetic couplings, the relaxation channels are segregated into two groups each with distinct time scales and as a result, a plateau in the decay of remanence appears. The Monte Carlo simulation suggests that inclusion of far-away neighbors does not modify our results significantly in one dimension. Thus the features should be characteristic of real chain structure. For an antiferromagnetic square lattice, a trimodal decay is observed if only nearest-neighbor interaction is considered. However, long-ranged interaction plays a very important role in $2 \mathrm{D}$ by smearing the plateaus into a continuous quasilogarithmic decay. For films with rectangular lattice, a transition between the bimodal decay and a quasilogarithmic decay is predicted when the ratio of length to width varies. Experimental possibility of observing the effect is discussed.

\section{THE MODEL}

Our model is based on the frozen ferrofluids studied in Ref. [5]. It consists of single domained particles with a size of $100 \AA$. Each particle has a magnetic moment for temperature below the Curie point which tends to align with the easy axis $[5,6,21,22]$. For simplicity, we assume that the applied magnetic field $H$ is along the easy axis, and the energy of each particle obeys $[7,15,17]$

$$
E=K V \sin ^{2} \theta-\mu H \cos \theta,
$$

where $K$ is the anisotropic energy constant, $V$ and $\mu$ the volume and the magnetic moment of the particle, respectively, and $\theta$ the angle between the magnetization and easy axis. The local minima of Eq. (1) are at $\theta=0$ and $\pi$. Thus the moment of each particle is equivalent to that of a twolevel system. As a result, the moment can also be represented by a variable $\sigma=\cos \theta$, taking only two values $\sigma=1,-1$ for $\theta=0, \pi$. Thermal flipping of magnetization between $\theta=0$ and $\pi$ across an energy barrier occurs at a frequency obeying Néel's formula [15]:

$$
W(\sigma \rightarrow-\sigma)=\nu_{0} \exp \left[-\frac{K V}{k_{B} T}\left(1+\frac{\mu H \sigma}{2 K V}\right)^{2}\right],
$$

where $W$ is the transition probability of flipping from one state to another in unit time, and $\nu_{0}$ is the characteristic frequency of the system at high temperature which has been suggested by Néel [15] and confirmed by experiment [5] as $10^{9} \mathrm{~Hz}$. Identifying $K V$ as $E_{b}, W$ has the exact form of Eq. (3). Here the effective magnetic field comes from the magnetic dipole-dipole interaction between particles:

$$
\vec{H}=3 \vec{r}_{i j}\left(\vec{r}_{i j} \cdot \vec{\mu}_{j}\right) / r_{i j}^{5}-\vec{\mu}_{j} / r_{i j}^{3},
$$

where $\vec{r}_{i j}$ is the vector of separation between particles $i$ and $j$.
First we consider a chain of $N$ equal-spaced particles with easy axes all parallel. The bond angle $\beta$, the angle between $\vec{r}_{i j}$ and the easy axes, takes two values, 0 and $\pi / 2$, which correspond to ferromagnetic and antiferromagnetic coupling, respectively [23]. Periodic boundaries are imposed and only the nearest-neighbor interaction is considered. Scaling time by $\nu_{0}^{-1}$ and the energy by anisotropy energy $K V$, we have

$$
W\left(\sigma_{i} \rightarrow-\sigma_{i}\right)=\exp \left[-T^{-1}\left(1+\frac{J \sigma_{i}\left(\sigma_{i-1}+\sigma_{i+1}\right)}{2}\right)^{2}\right],
$$

where $\quad T=k_{B} T_{\text {unscaled }}(K V)^{-1}$ and $J=2 \mu^{2}\left(K V r^{3}\right)^{-1}$, $-\mu^{2}\left(K V r^{3}\right)^{-1}$ for $\beta=0, \pi / 2 . r$ is the separation between particles. For a one-dimensional system with Ising-like spin variable and nearest-neighbor interaction, it has been shown by Glauber that the general form of transition probability is [10]

$$
\begin{aligned}
W\left(\sigma_{i} \rightarrow-\sigma_{i}\right)= & \frac{1}{2} \alpha\left\{1+\delta \sigma_{i-1} \sigma_{i+1}\right. \\
& \left.-\frac{1}{2} \gamma(1+\delta) \sigma_{i}\left(\sigma_{i-1}+\sigma_{i+1}\right)\right\},
\end{aligned}
$$

where $\gamma=\tanh (2 J / T)$ is determined from detailed balance condition. $\alpha$ and $\delta$, however, cannot be determined without further specifying the system. In Eq. (8), if we define

$$
\begin{gathered}
\alpha=\exp (-1 / T)\left[1+\exp \left(-J^{2} / T\right) \cosh (2 J / T)\right], \\
\delta=\frac{\exp \left(-J^{2} / T\right) \cosh (2 J / T)-1}{\exp \left(-J^{2} / T\right) \cosh (2 J / T)+1},
\end{gathered}
$$

then Eqs. (7) and (8) are the same. Glauber obtained analytical solution of Eq. (8) only for $\delta=0$; the resulting decay of the total magnetization is a simple exponential [10]. In the present case, $\delta$ is nonzero.

The dynamic evolution of the system is governed by a stochastic master equation:

$$
\begin{aligned}
\frac{d}{d t} p\left(\sigma_{1} \cdots \sigma_{N}, t\right)= & -\sum_{i} W\left(\sigma_{i} \rightarrow-\sigma_{i}\right) p\left(\sigma_{1} \cdots \sigma_{i} \cdots \sigma_{N}, t\right) \\
& +\sum_{i} W\left(-\sigma_{i} \rightarrow \sigma_{i}\right) \\
& \times p\left(\sigma_{1} \cdots-\sigma_{i} \cdots \sigma_{N}, t\right)
\end{aligned}
$$

where $p\left(\sigma_{1} \cdots \sigma_{N}, t\right)$ is the probability that the spins take on the set of values $\left(\sigma_{1} \cdots \sigma_{N}\right)$ at time $t$. With $\delta$ nonzero, the analytic solution of Eq. (10) is impossible for arbitrary $N$. For small $N$, however, it is possible to solve its eigenmodes numerically. To eliminate nonmagnetic modes which describe deviation from equilibrium without change of magnetization, we observe from Eq. (10) that

$$
\begin{aligned}
\frac{d}{d t}\left\langle\sigma_{i_{1}} \cdots \sigma_{i_{k}}\right\rangle= & -2\left\langle\sigma _ { i _ { 1 } } \cdots \sigma _ { i _ { k } } \left[ W\left(\sigma_{i_{1}} \rightarrow-\sigma_{i_{1}}\right)\right.\right. \\
& +\cdots+W\left(\sigma_{i_{k}}\right. \\
\rightarrow & \left.\left.\left.-\sigma_{i_{k}}\right)\right]\right\rangle,
\end{aligned}
$$


where $\langle A\rangle=\sum_{\sigma_{1} \cdots \sigma_{N}} A(\sigma) p\left(\sigma_{1} \cdots \sigma_{N}, t\right)$ denotes the expectation value of $A$ at time $t$. In particular, magnetization is $M=\sum_{i=1}^{N}\left\langle\sigma_{i}\right\rangle$. Using Eq. (11), one-spin functions such as $M=\sum_{i=1}^{N}\left\langle\sigma_{i}\right\rangle$ will be coupled to three-spin functions such as $G_{31}=\sum_{i=1}^{N}\left\langle\sigma_{i-1} \sigma_{i} \sigma_{i+1}\right\rangle$, which will in turn be coupled to other spin correlation functions. The process will be truncated automatically as the correlation functions approach the size of the chain. In this way, a much smaller eigensystem is obtained. For $N=10$, we have a $34 \times 34$ matrix, compared to the otherwise $1024 \times 1024$ matrix from the original master equation. The complete sets of matrix elements are obtained in this way for $N \leqslant 10$.

Systems of longer chains $(N \sim 10000)$ and film geometry can be explored by Monte Carlo simulation. The effect of long-ranged interaction is also investigated. For film structure, we consider a rectangular lattice with easy axis of all particles normal to the plane. This introduces an antiferromagnetic coupling among particles. We adopt the algorithm 2 of Binder [24], which may be summarized as follows. For an assembly of $N$ particles, the transition rate of the system $R$ is defined as the probability per unit time that a flipping occurs, which is equal to the sum of transition rate of individual particle $W\left(\sigma_{i}\right)$ :

$$
R=\sum_{i} W\left(\sigma_{i}\right)
$$

The probability that the earliest flipping occurs between $t$ and $t+d t$ for the $i$ th spin is

$$
P_{i}(t) d t=W\left(\sigma_{i}\right) \exp (-R t) d t
$$

In practice, this is realized through the following steps.

(1) Set $t=0$.

(2) Sample $t_{i}$ for each spin according to the distribution $W(i) \exp [-W(i) t]$.

(3) Set $t=t+\min \left\{t_{i}\right\}$, in which a particular spin is chosen according to $t_{\nu}=\min \left\{t_{i}\right\}$.

(4) Spin $\nu$ is allowed to flip.

(5) Steps (2)-(4) are repeated.

The algorithm is very efficient for dynamic process involving time scales much longer than the basic unit scale $\nu_{0}^{-1}$.

All our calculations involve the remanent decay [25]. The spins are initially up in the presence of a saturation field. The field is removed at $t=0$ and the decay begins. The normalized magnetization is

$$
m(t)=\frac{M(t)}{M(0)}=N^{-1} \sum_{i=1}^{N}\left\langle\sigma_{i}\right\rangle=\sum_{k=1}^{l} w_{k} \exp \left(-\lambda_{k} t\right),
$$

where $\lambda_{k}$ is the eigenfrequency of channel $k$ and for finite system the integral over $\tau$ in Eq. (1) is replaced by a summation over discrete channel $k$. The nonlinear relaxation time $\tau_{\mathrm{nl}}$ is defined as $[11,12]$

$$
\tau_{\mathrm{nl}}=\int_{0}^{\infty} d t m(t)=\sum_{k=1}^{l} w_{k} \tau_{k}
$$

where $\tau_{k}=\lambda_{k}^{-1}$ is the relaxation time of channel $k$.

\section{RESULTS AND DISCUSSION}

\section{A. Analytical results for $N=1,2,3$}

For the cases of $N=1,2$, time decays are easily calculated from Eqs. (5), (9), and (10) and are shown to be simple exponential: $m(t)=e^{-\lambda t}$. For $N=1, \lambda=2 e^{-1 / T}$. For $N=2$, $\lambda=2 \exp \left[-T^{-1}(1+J / 2)^{2}\right]$. Here the interaction simply increases the relaxation time $\tau=\lambda^{-1}$ for ferromagnetic coupling $J>0$ and decreases the relaxation time $\tau$ for antiferromagnetic coupling $J<0$. For $N \geqslant 3$, the dynamics is complex. We begin with the case of $N=3$. The case can be reduced to a $2 \times 2$ eigenproblem and is exactly solvable. The solution exhibits important features of bimodal decay which persist even for large $N$.

For three particles, uses of Eq. (11) on $\left\langle\sigma_{i}\right\rangle$ and $\left\langle\sigma_{i-1} \sigma_{i} \sigma_{i+1}\right\rangle$ produce two coupled equations relating $M$ $=\Sigma_{i}\left\langle\sigma_{i}\right\rangle$ and $G=\Sigma_{i}\left\langle\sigma_{i-1} \sigma_{i} \sigma_{i+1}\right\rangle$ :

$$
\frac{d}{d \alpha t}\left(\begin{array}{c}
M \\
G
\end{array}\right)=\left(\begin{array}{cc}
-1+\gamma(1+\delta) & -\delta \\
3[\gamma(1+\delta)-\delta] & -3
\end{array}\right)\left(\begin{array}{c}
M \\
G
\end{array}\right) .
$$

Eigenvalues and eigenvectors can be easily computed for this set of equations. To determine $w_{k}$ for the remanent decay, we note that at $t=0,\left.\left(\begin{array}{c}M \\ G\end{array}\right)\right|_{t=0}=\left(\begin{array}{l}3 \\ 3\end{array}\right)$. This can be expressed as a linear combination of right eigenvectors. In this way, we find

$$
\begin{gathered}
\lambda_{1,2}=\alpha / 2[4-\gamma(1+\delta) \pm U], \\
w_{1,2}=\frac{1}{2}\left\{1 \pm U^{-1}[2 \delta-2-\gamma(1+\delta)]\right\} \\
U=\sqrt{[4-\gamma(1+\delta)]^{2}-12(1-\gamma)\left(1-\delta^{2}\right)} .
\end{gathered}
$$

Now suppose that interaction $|J|$ is larger than thermal energy but both are smaller than anisotropic energy $K V$. Then we have $2|J| / T>1, \exp (-2|J| / T) \ll 1$, and $\exp \left(-J^{2} / T\right) \sim 1$. Using Eq. (9) and to the leading term of $O[\exp (-2|J| / T)]$, we have for $J>0$,

$$
\begin{gathered}
\lambda_{1} \approx \exp \left[-(1-J)^{2} / T\right], \\
\lambda_{2} \approx 12 \exp [-(1+4 J) / T], \\
w_{1} \approx 0, \\
w_{2} \approx 1,
\end{gathered}
$$

and for $J<0$,

$$
\begin{gathered}
\lambda_{1} \approx 3 \exp \left[-(1-|J|)^{2} / T\right], \\
\lambda_{2} \approx 4 \exp (-1 / T), \\
w_{1} \approx \frac{2}{3}-\frac{4}{9} \exp \left[-\left(2|J|-J^{2}\right) / T\right], \\
w_{2} \approx \frac{1}{3}+\frac{4}{9} \exp \left[-\left(2|J|-J^{2}\right) / T\right] .
\end{gathered}
$$

An important difference is observed in dynamics between ferromagnetic and antiferromagnetic coupling from Eqs. (18a) and (18b). For $J>0$, the slower channel has nearly $100 \%$ weight and is thus dominant. The corresponding decay can be approximated as simple exponential with a relaxation time $\tau_{2}=\lambda_{2}^{-1}$ which increases exponentially with $J$. On the 


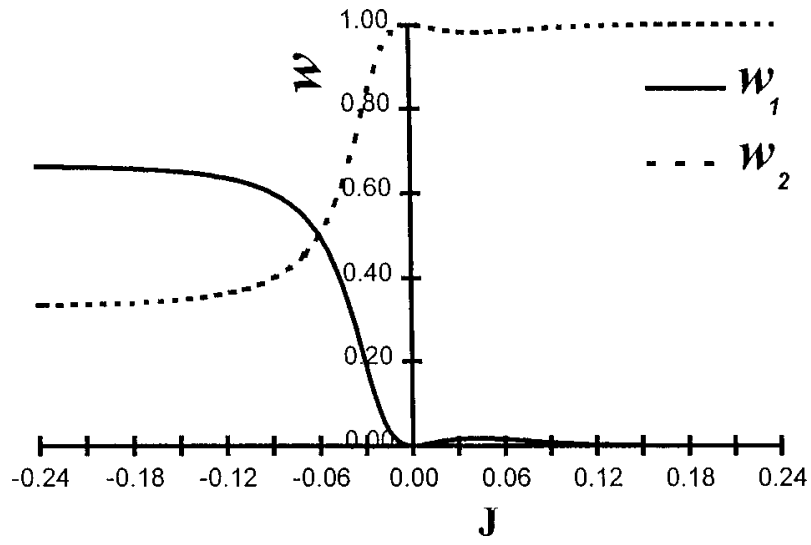

FIG. 1. The distribution of two relaxation channels $w_{1}$ and $w_{2}$ as a function of coupling strength $J(J>0$, ferromagnetic coupling and $J<0$, antiferromagnetic coupling) for $N=3$, and the blocking temperature $T_{B}=0.04$.

other hand, for $J<0$, the two channels of decay are comparable and the dynamics will be characterized by two time scales $\tau_{1}$ and $\tau_{2}$. While the shorter time $\tau_{1}$ decreases exponentially with increasing $|J|$, the longer time $\tau_{2}$ is nearly fixed. At large $|J|, w_{1}$ and $w_{2}$ approach their asymptotic values of $\frac{2}{3}$ and $\frac{1}{3}$, respectively. In this limit, $\tau_{2} \gg \tau_{1}$ and the nonlinear relaxation time is [see Eq. (15)]

$$
\tau_{\mathrm{nl}} \approx w_{2} \tau_{2} \approx(1 / 12) \exp (1 / T)
$$

independent of $J$. In Fig. $1, w_{1}$ and $w_{2}$ are plotted as a function of $J$ at $T_{B}$, the blocking temperature of a single magnetic dipole. $\left(T_{B}=0.04\right.$, or in real dimension $K V / k_{B} T=$ 25.) From Fig. 1, we see that the dominance of the slower channel over the faster channel is established for the entire range of ferromagnetic coupling $J>0$.

\section{B. Numerical results for $3<N<10$}

The above features are typical of the dynamics for chain structures. For $N$ larger than 3 and for large antiferromagnetic couplings, the bimodal character remains. By using Eq. (11) for various correlation functions, $\tau_{i}=\lambda_{i}^{-1}$ and $w_{i}$ can be determined numerically by solving the matrix for eigenvalues. For $3<N<10$, the time decay of the remanence is trivial for the ferromagnetic coupling - the dominant channel accounts for over $96 \%$ of the total weight. This results from the fact that, at the early stage, the remanence relaxes through the slowest channel, i.e., the longest time scale. In contrast, for antiferromagnetic coupling, the shortest time scale is responsible for the initial decay and a two-channel decay is observed.

A spectrum for antiferromagnetic coupling at $N=10$ and different $J$ is shown in Fig. 2. A prominent feature of the spectrum is its two distinct channels - the fast and the slow modes. With increasing $|J|$, the fast modes shift towards small $\tau$ while the slow modes are nearly fixed in weight and relaxation time $\tau$, insensitive to the variation of $|J|$. This indicates that most spin flips occur around two distinct time scales: $\tau_{1}<\tau_{2}$ and a plateau in between. In Fig. 3, decays of remanent magnetizations for different $N$ at $T=0.04$ and $J$ $=-0.32$ are plotted. After the sudden drop at the early time, each system retains a finite residual magnetization $m_{r}$,
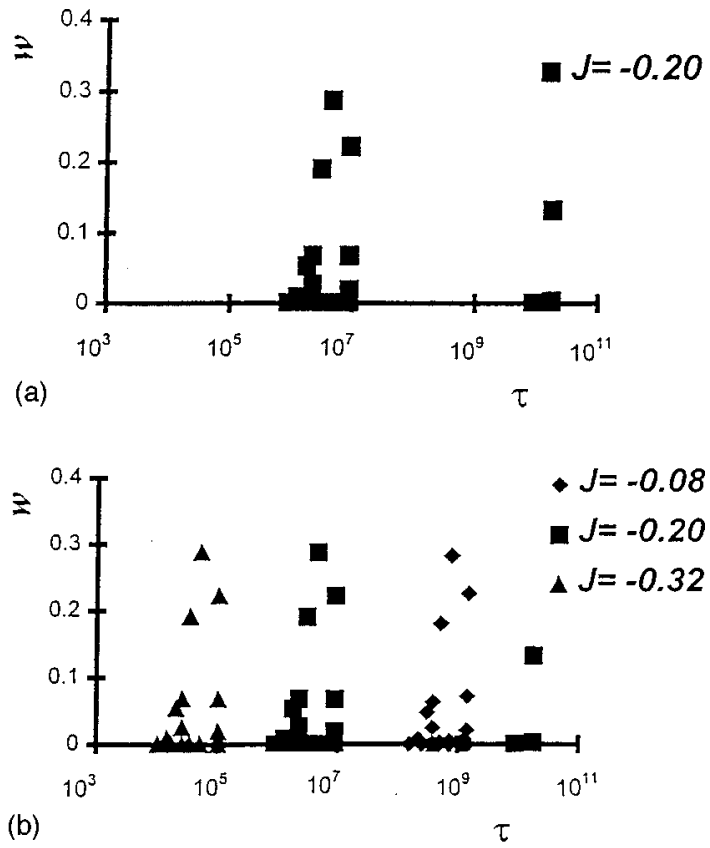

FIG. 2. The distribution of relaxation channels versus $\tau_{i}$ for $N$ $=10$ and $T=0.04$. (a) A spectrum showing two relaxation channels at $J=-0.2$. (b) As $|J|$ increases from 0.08 to 0.32 , the fast channels consistently shift towards small relaxation times while the slow channels appear to be fixed. Note that at the right end of the spectrum (slow modes), three types of symbols representing different $J$ 's overlap with each other and are difficult to distinguish.

which does not decay until a much later time that represents the free relaxation of a single particle. In large coupling limit, similar to $N=3$, we have $\tau_{2} \gg \tau_{1}$ and the nonlinear relaxation time is

$$
\tau_{\mathrm{nl}} \approx m_{r} \tau_{2}
$$

independent of $J$ and solely determined by the slower time scale. The only exception is for $N=4$ where only a group of two fast modes is found.

We interpret the bimodal feature in the following way. There are two distinct time scales, $\tau_{1} \ll \tau_{2}$, where $\tau_{1}$ corresponds to the flip time of a spin under the influence of the

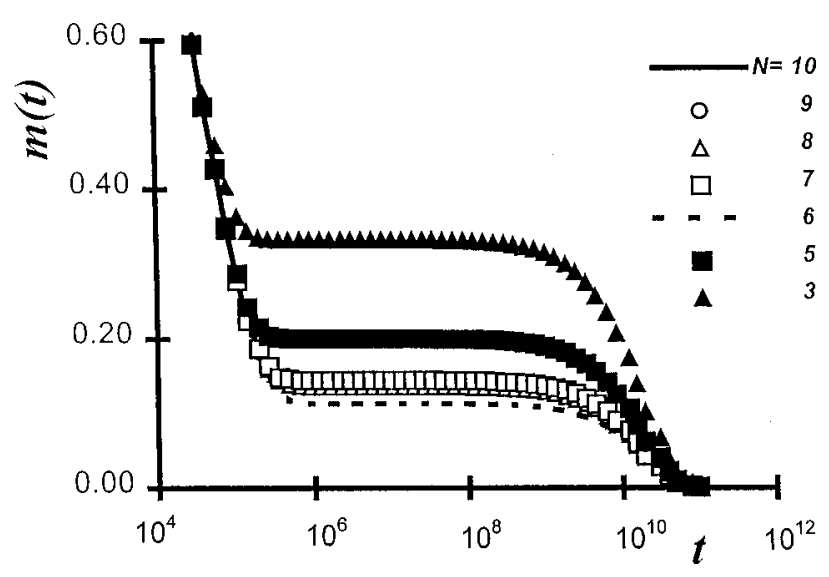

FIG. 3. Time decays of remanence for different $N$ at $T=0.04$ and $J=-0.32$. A plateau in $m(t)$, that extends to four decades, exists in all cases. Note the curves are nearly indistinguishable from each other for $N \geqslant 8$. 
TABLE I. The plateau height $m_{r}$ at finite $N$. The $m_{r}^{*}$ are evaluated by spectrum decomposition at $T=0.04$ and $J=-0.32$.

\begin{tabular}{lccccccccc}
\hline \hline$N$ & 3 & 5 & 6 & 7 & 8 & 9 & 10 & 11 & 12 \\
\hline$m_{r}$ as fraction & $1 / 3$ & $1 / 5$ & $1 / 9$ & $1 / 7$ & $2 / 15$ & $11 / 81$ & $71 / 525$ & $67 / 495$ & $1151 / 8505$ \\
$m_{r}$ 's value & 0.33333 & 0.20000 & 0.11111 & 0.14286 & 0.13333 & 0.13580 & 0.13524 & 0.13535 & 0.13533 \\
$m_{r}^{*}$ & 0.33333 & 0.20000 & 0.11111 & 0.14286 & 0.13333 & 0.13580 & 0.13524 & & \\
\hline \hline
\end{tabular}

interaction and $\tau_{2}$ is the relaxation time of the free particle at blocking temperature. Spins which can flip at time scale around $\tau_{1}$ are considered as superparamagnetic while spins which can flip at time longer than $\tau_{2}$ are considered as blocked. So a spin is superparamagnetic only if the two neighboring spins are parallel to it. As a result, in the time window of $\tau_{1}$, the flipping is completely irreversible. For any $N$, we start with the spin-all-up configuration and proceed every parallel flipping event with equal probability until all spins are blocked inside the time window of $\tau_{1}$. The magnetization of the blocked state, divided by $N$, gives the value of $m_{r}$. Following examples demonstrate how $m_{r}$ is determined (we represent a chain of spins by $\uparrow$ - spin up and $\downarrow$-spin down). For a 1D chain, $m_{r}$ cannot exceed $\frac{1}{3}$, the value corresponding to a configuration of repetition of trimer such as $\uparrow \uparrow \downarrow$. Keeping the periodic boundary condition in mind, we realize that every spin in this configuration is blocked and this is exactly the case for $N=3$.

For $N>3$, due to the stochastic nature of the flipping, $m_{r}$ is reduced and approaches to a convergent value when $N$ $\sim 10$. To illustrate the method, the cases of $N=4,6$, and 9 are presented (recall the periodity of the boundary):

$$
\begin{gathered}
\uparrow \uparrow \uparrow \uparrow \Rightarrow \uparrow \uparrow \downarrow \uparrow \Rightarrow \downarrow \uparrow \downarrow \uparrow \Rightarrow \text { blocked, } \\
\uparrow \uparrow \uparrow \uparrow \uparrow \uparrow \Rightarrow \uparrow \uparrow \downarrow \uparrow \uparrow \uparrow \Rightarrow \frac{1}{3} \uparrow \uparrow \downarrow \uparrow \uparrow \downarrow+\frac{2}{3} \downarrow \uparrow \downarrow \uparrow \downarrow \uparrow \Rightarrow \text { blocked, }
\end{gathered}
$$

$$
\begin{aligned}
& \uparrow \uparrow \uparrow \uparrow \uparrow \uparrow \uparrow \uparrow \uparrow \Rightarrow \uparrow \uparrow \uparrow \uparrow \downarrow \uparrow \uparrow \uparrow \uparrow \\
& \Rightarrow \frac{1}{3} \uparrow \uparrow \uparrow \downarrow \uparrow \downarrow \uparrow \uparrow \uparrow+\frac{1}{3} \uparrow \uparrow \downarrow \uparrow \uparrow \downarrow \uparrow \uparrow \uparrow \\
&+\frac{1}{3} \uparrow \uparrow \downarrow \uparrow \uparrow \uparrow \downarrow \uparrow \uparrow \\
& \Rightarrow \frac{1}{3} \uparrow \downarrow \uparrow \downarrow \uparrow \downarrow \uparrow \uparrow \downarrow+\frac{1}{9} \uparrow \uparrow \downarrow \uparrow \uparrow \downarrow \uparrow \uparrow \downarrow \\
&+\frac{2}{9} \downarrow \uparrow \downarrow \uparrow \uparrow \downarrow \uparrow \downarrow \uparrow+\frac{1}{3} \downarrow \uparrow \downarrow \uparrow \downarrow \uparrow \downarrow \uparrow \uparrow \\
&= \text { blocked. }
\end{aligned}
$$

These give $m_{r}=\frac{1}{9}$ and $\frac{11}{81}$ for $N=6$ and 9. Equation (21a) also shows that $m_{r}=0$ for $N=4$, an anomalous case with no plateau character. Results for different $N$ were compiled in Table I for $N$ up to 12. Also shown in Table I is $m_{r}^{*}$, the value of $m_{r}$ determined by the spectrum decomposition at $T=0.04$ and $J=-0.32$ by summing the distribution weights of the slow channels: $m_{r}=\Sigma_{\text {slow modes }} w_{i}$. Both methods give identical result of $m_{r}$ to the displayed accuracy. This intuitive diagrammatic approach also suggests that spins with one neighboring spin up and one neighboring spin down, experiencing no local field, decay freely only at time scale $\tau_{2}$.
Figure 3 suggests that results for large $N$ might converge to a unique curve, an important implication for macroscopic systems. The $m_{r}$ in Table I exhibits an oscillatory convergence with $N$. From this convergent character, it is concluded that the value of $m_{r}$ for infinite long chain is between 0.13533 and 0.13535 . At large coupling limit, this value is solely determined by the statistics of irreversible flipping according to a simple mathematical rule and is independent of physical parameters.

\section{Monte Carlo simulations}

\section{Antiferromagnetic chain}

In order to decide the effect of long-range dipole interaction from all neighbors on the results from the preceding section, in which only the nearest neighbors are included, and to confirm the convergence of the decay curves, Monte Carlo simulation of remanence decay was performed for much larger systems. Although the discrete spectrum is lost, a meaningful comparison can be made with previous analysis for finite $N$.

In Fig. 4 simulated remanence decays for $N=10000$ at $J=-0.32$ and blocking temperature of $T=0.04$ are shown along with numerical calculation of $N=10$. Also shown is the simulated decay with the long-range interaction considered for all spins. For nearest-neighbor interaction, the Monte Carlo simulation and analytical calculation for $N$ $=10$ show remarkable agreement $\left(m_{r} \approx 0.135\right)$. Inclusion of

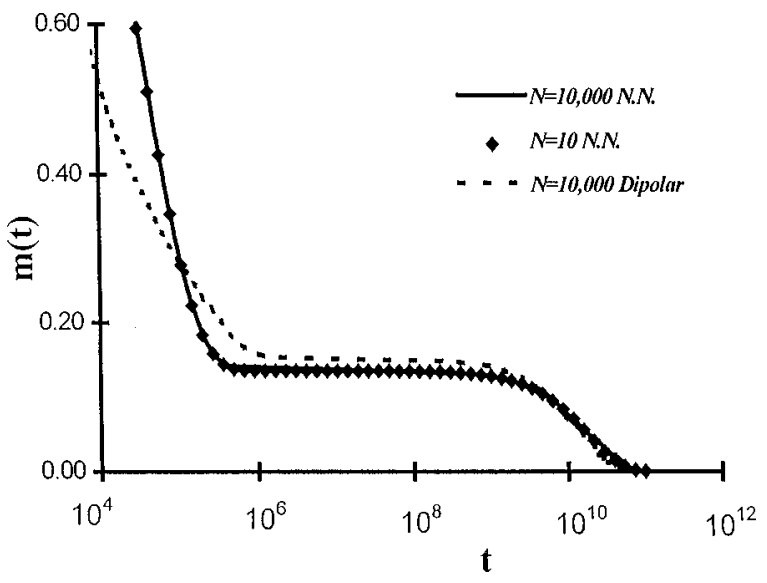

FIG. 4. The decay of remanence from Monte Carlo simulation for a chain with $N=10000, T=0.04$, and $J=-0.32$. N.N. includes nearest-neighbor interaction only while dipolar interaction includes all the long-range contributions. Also included is the $N=10$ result from direct calculation of eigenmodes. For the N.N. case, the results of $N=10$ and 10000 show perfect agreement. Inclusion of dipolar contribution beyond nearest neighbor produces minor modification in $1 \mathrm{D}$. 


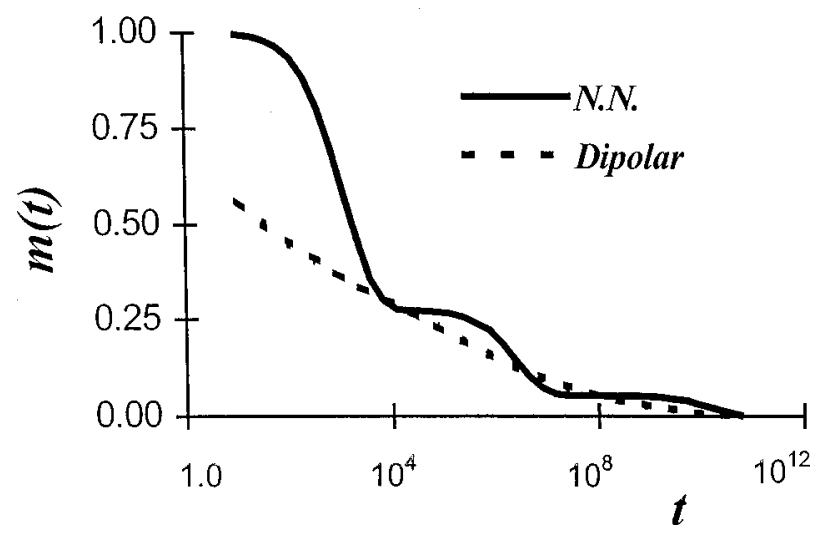

FIG. 5. The decay of remanence from Monte Carlo simulation for a $40 \times 40$ square lattice. $T=0.04$ and $J=-0.22$. For nearestneighbor approximation (N.N.), two plateaus (trimodal) are displayed with heights 0.27 and 0.06 . The heights are determined from the plotting data. Dipolar contribution beyond nearest neighbor is much more important in $2 \mathrm{D}$ and the resulting decay is quasilogarithmic, in accordance with the mean-field prediction.

far-away-neighbor contributions produces minor modification-the curve shifts towards small $\tau$ at early time and $m_{r}$ is slightly larger. This indicates that the contribution beyond the nearest neighbor is less important in 1D dynamics and justifies our use of nearest-neighbor approximation. Snapshots taken from pieces of the chain within the time range of the plateau confirm the previously mentioned picture: The spins that have not yet decayed (the residual spins) are caught between antiparallel spins and experience no driving force even though the average field, or the mean field, is not zero at the time.

The mean-field theory for $J<0$ predicts a quasilogarithmic remanent decay. For a system to be quasilogarithmic over decades, $w(\tau)$ must extend over decades and be uniformly distributed over logarithmic scale of $\tau$ [26], in contrast to the composition of $w(\tau)$ of the above model. This disagreement is due to the low dimensionality of the model and the breakdown of the mean-field assumption.

\section{2D film with perpendicular anisotropy}

To study the effect of dimensionality, time decay of remanence for a $2 \mathrm{D}$ antiferromagnetic film is simulated. The geometric arrangement is such that all particles embedded in the film have anisotropic axis normal to the plane. This is the geometry used in most particulate normal recording media [17-20]. Figure 5 shows simulated decays for a $40 \times 40$ square lattice with $J=-0.22$ and blocking temperature $T$ $=0.04$. With the nearest-neighbor approximation, the decay can be characterized by a trimodal behavior (that is, two plateaus) with $\tau_{1} \ll \tau_{2} \ll \tau_{3}$ and $w_{1} \sim 0.73, w_{2} \sim 0.21$, and $w_{3}$ $\sim 0.06$. For each spin with four nearest neighbors, these time scales are easily identified with the three stages of relaxations corresponding to different neighboring configurations: (1) relaxation at $\tau_{1}$ is for spins with four neighbors parallel, (2) the one at $\tau_{2}$ is for spins with three neighbors parallel and one antiparallel, and (3) free relaxation at $\tau_{3}$ is for spins with two neighbors parallel and two antiparallel. However, the long-ranged coupling plays a very important role in $2 \mathrm{D}$ be-

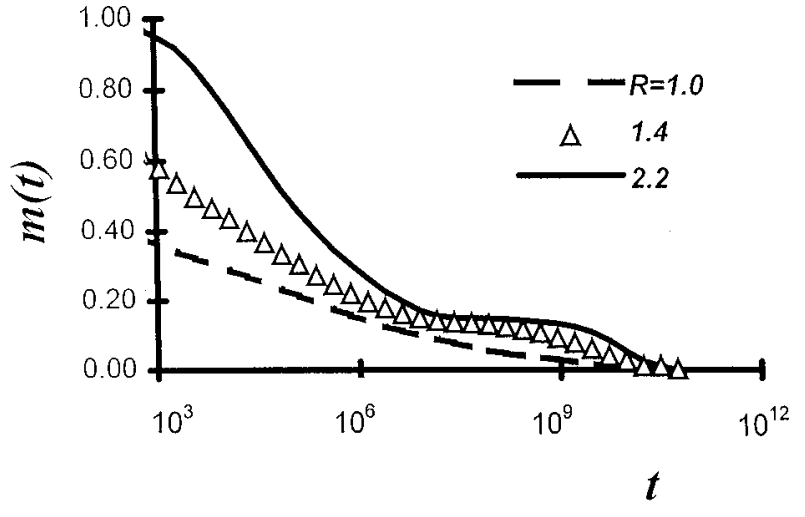

FIG. 6. Decays of remanence from Monte Carlo simulation for a $40 \times 40$ stretchable rectangular lattice with a length-to-width ratio $R$. $T=0.04$ and $J=-0.22$. Increasing $R$ from 1 to 2.2 , a transition from quasilogarithmic to bimodal decay is observed.

cause a larger number of configurations leads to more relaxation channels. This will result in a continuous quasilogarithmic decay in agreement with the mean-field theory, as shown in Fig. 5. Inclusion of a $7 \times 7$ array of neighbors has shown convergence in this case and the quasilogarithmic law is exhibited over nine decades.

Finally we consider a special geometry which allows a transition of dynamics between quasilogarithmic and bimodal. The construction uses a stretchable rectangular lattice with a length-to-width ratio $R[21]$ ( $R=1$ for square lattice). For $R>1$, the effective dimensionality is reduced with an unbalanced coupling. Figure 6 shows remanent decays for a $40 \times 40$ lattice with $J=-0.22$ and blocking temperature $T$ $=0.04$ for different $R$. For $R=1$, the decay is quasilogarithmic. Increasing $R$ to 2.2 , the decay shows bimodal character with a decayless plateau extending about three orders of magnitude, separating the fast and slow decays. The time distribution of the fast decay is broader as a result of the lateral coupling.

For monodispersed spherical cobalt particle $H_{K}=3 \mathrm{kOe}$ and a typical diameter $D=64 \AA$, the blocking temperature $T=0.04$ corresponds to a temperature of $84 \mathrm{~K}$ while $J=$ -0.22 corresponds to an interparticle spacing of $85 \AA$. For film morphology with magnetic micropore deposition where the reversal mechanism is known to be incoherent [19], smaller $T$ should be used due to the overestimate of $K$. Other realistic complications may also arise, for example, polydispersity, geometric correction, and so on. However, the feasibility of such experimental observation is possible. So far only quasilogarithmic time dependence has been reported on systems used as recording media $[16,18]$.

\section{CONCLUSIONS}

Effects of interaction on the dynamics of dipolar systems are studied by analytical, numerical, and the Monte Carlo simulation. The dramatic difference was found between the ferromagnetic and the antiferromagnetic couplings in onedimensional systems. For ferromagnetic interaction, the time decay of the remanent magnetization is essentially exponential, while for antiferromagnetic interaction, two distinct time scales were found; the plateau between the two times corre- 
sponds to the blocking of spins. The bimodal feature was found to cross over to a quasilogarithmic decay when the dimensionality is increased. There is a similarity between the current model and the mode-coupling theory [27] in which decoupling of relaxation channels was also found.

We would like to point out that a notable difference exists between the present model and the particular choice of Glauber's solution. With $\delta=0$, the ratio of antiferromagnetic relaxation time $\tau_{1}$ and the free decay time $\tau_{2}$ is $\tau_{1}^{-1}: \tau_{2}^{-1}$ $=[1+\tanh (2|J| / T)]: 1$, so that for any value of $|J|$, the ratio is between 1 and 2 and the bimodal segregation is not pos- sible, in contrast to the case of Eq. (7) where $\tau_{1}$ and $\tau_{2}$ can differ by many orders of magnitude for $|J|>T$.

\section{ACKNOWLEDGMENTS}

This work was inspired by the experiment by Zhang, Boyd, and Luo [5]. We are grateful for useful discussions with Dr. A. Cebers, Jinlong Zhang, and Tengda Du. This work was financially supported by the NSF Young Investigator Program (W. L.).
[1] For review on glasses, see, e.g., C. A. Angell, in Proceedings of the Workshop on Relaxations in Complex Systems, Blacksburg, VA, 1983, edited by K. L. Ngai and G. B. Wright (U.S. Office of Naval Research, Arlington, VA, 1984).

[2] N. Menon and S. R. Nagel, Phys. Rev. Lett. 74, 1230 (1995), and references therein.

[3] For a review on spin glasses, see K. Binder and A. P. Young, Rev. Mod. Phys. 58, 801 (1986).

[4] Weili Luo et al., Phys. Rev. B 41, 4465 (1990); Weili Luo et al., J. Phys. (Paris), Colloq. 49, C8-1151 (1988), and references therein.

[5] J. Zhang, C. Boyd, and Weili Luo, Phys. Rev. Lett. 77, 390 (1996).

[6] Weili Luo et al., Phys. Rev. Lett. 67, 2721 (1991).

[7] S. Dattagupta, Relaxation Phenomena in Condensed Matter Physics (Academic, London, 1987).

[8] K. L. Ngai, Comments Solid State Phys. 9, 127 (1979); 9, 141 (1979).

[9] R. G. Palmer et al., Phys. Rev. Lett. 53, 958 (1984).

[10] R. Glauber, J. Math. Phys. (N.Y.) 4, 294 (1963).

[11] E. Stoll, K. Binder, and T. Schneider, Phys. Rev. B 8, 3266 (1973).

[12] H. G. E. Hentschel, Z. Phys. B 37, 243 (1980).

[13] M. Suzuki and R. Kubo, J. Phys. Soc. Jpn. 24, 51 (1968).
[14] H. Yahata, J. Phys. Soc. Jpn. 30, 657 (1971).

[15] L. Néel, C. R. Acad. Sci. 228, 664 (1949); Rev. Mod. Phys. 25, 293 (1953).

[16] D. K. Lottis, E. D. Dahlberg, J. A. Christner, J. I. Lee, R. L. Peterson, and R. M. White, J. Appl. Phys. 63, 2920 (1988).

[17] D. K. Lottis, R. M. White, and E. D. Dahlberg, Phys. Rev. Lett. 67, 362 (1991).

[18] A. Lyberatos et al., IEEE Trans. Magn. MAG-26, 222 (1990).

[19] A. Lyberatos, R. W. Chantrell, E. R. Sterringa, and J. C. Lodder, J. Appl. Phys. 70, 4431 (1991).

[20] E. P. Wohlfarth, Phys. Lett. 70A, 489 (1979).

[21] I. Klik and C. R. Zhang, Phys. Rev. B 52, 3540 (1995).

[22] I. Klik, C. R. Chang, and J. S. Yang, J. Appl. Phys. 76, 6588 (1994).

[23] W. Chen, S. Zhang, and H. N. Bertram, J. Appl. Phys. 71, 5579 (1992).

[24] K. Binder, Monte Carlo Methods in Statistical Physics, 2nd ed. (Springer-Verlag, Berlin, 1979).

[25] R. W. Chantrell, A. Lyberatos, and E. P. Wohlfarth, J. Phys. F 16, L145 (1986).

[26] E. D. Dahlberg et al., J. Appl. Phys. 76, 6396 (1994).

[27] W. Götze and L. Sjögren, J. Phys. C 17, 5759 (1984); Z. Phys. B 65, 415 (1987). 